\title{
UNEMPLOYMENT AMONG WOMEN OVER FORTY
}

$\mathrm{U}^{\mathrm{N}}$ NLIKE many societies where the bulk of the economic load is carried by women, Western culture in general and British society in particular have inherited a tradition that opposes the gainful employment of women. To the employer women workers present problems different from those of men. Employers declare that if a woman is single she tends to be temporary, filling in time until she is married; and that if she is married she has domestic responsibilities which may make her unreliable as a worker. The sickness-rate is said to be higher among women, though here it is difficult to get reliable statistics. A woman is also said to age sooner than a man, though her average expectation of life is greater. The precise accuracy of these statements is not known; the fact that they are widely believed creates an employment complication of considerable significance.

Four surveys of unemployment among women over forty years of age in the Southampton area have been made by Miss A. F. Young of the University of Southampton (Personnel Management, 40, No. 344 ; June 1958). The total number of women employed in the area has risen from about 19,000 in 1951 to more than 23,000 in 1956, and total unemployment has fluctuated between 363 in 1954, and 472 in 1953 , or about 2 per cent of all women in work or seeking it. Of the women unemployed, the number over forty has varied between 88 in 1951 and 204 in 1953, that is, between about a quarter and a half of the total, though in the last two surveys the proportion has been about a third. The figures show that the number of unemployed older workers remains obstinately high even when, as in 1954, unemployment was at its lowest. This leads to the assumption that a hard core of unemployment exists among those over forty.

Nearly half the number were found to be married women, with presumably a husband and a home. Of the rest the proportion of single and widowed fluctuated, but averaged a little more than 25 per cent each of the total. It was found, however, that unemployed single women over forty sometimes reached as much as 30 per cent of the whole (1954). When those over forty were analysed according to their age, it was found that more than half were fifty or older ( 57 per cent, in 1956); and getting a job becomes more difficult the older a woman grows.
The 'social' reason for discouraging the older woman to take gainful employment is her responsibility to dependent children; this is the reason why married women so often interrupt their employment for a number of years. The surveys disclosed that, of the 68 married and 43 widows over forty, only 19 had any dependent children and, of these, 7 were over fifty, the rest being under fifty. This was the position in 1956, and the figures for 1954 show a similar situation. It became clear that the demands of young children are not, on the whole, a complication to be contended with in the employment of women over forty.

It is often alleged that the majority of older women are not available for full-time work, either because their health is not good enough or their domestic responsibilities make it impossible or, having left industry for a number of years, they cannot face the discipline and strain of full-time work. The facts do not support this contention; nearly two-thirds of the women in two of the surveys have asked for full-time employment. Full-time work is more possible for those under fifty than later. Against this survey it was found that, of the small number of women over sixty looking for work, nearly all wanted it full-time.

Allied to the older woman's availability for work is her reason for seeking it. Three main reasons were given: that the wage would be her sole income; that she wished to supplement her own resources; or that she needed to supplement the family income. A fourth category of "other reasons" included the need for companionship, inability to break the habit of going out to work, and other non-financial causes.

From the evidence of these surveys there is some substance in the saying "too old at forty" where women are seeking gainful employment. If this notion is to be broken at all, and the reservoir of available labour exploited to the full for the increasing productivity of our society, then some positive steps seem to be necessary. Apart from extending the number of employers who adapt their works to meet the needs of women, like part-time work of various kinds, they might include propaganda about the advantage of maturity, training, and direct recruitment into domestic and catering trades where there are many vacancies and where many older women could usefully be absorbed.

\section{SOUTHERN STARS OF HIGH VELOCITY}

$\mathrm{A}^{\mathrm{s}}$ S might be expected from the fact that observatories of the southern hemisphere are of comparatively recent origin and have fewer astronomers and telescopes at their disposal than those in the northern hemisphere, the data for large groups of southern stars are by no means complete. This lack of data is particularly noticeable in the determination of radial velocities, a survey of which was completed for stars brighter than magnitude $\mathbf{5 \cdot 5}$. After the publication of Miss Roman's "Catalogue of High-Velocity Stars", which included stars north of Dec. $-20^{\circ}$ and brighter than visual magnitude $9 \cdot 5$, it was considered that a complementary list of southern high-velocity stars would be of great interest, but, owing to the lack of observational data, it will probably be many years before a survey similar to Miss Roman's will be completed. With the object of serving as a basis for future observations the present finding list, including all available information, has been published*.

As the criteria for inclusion in the list are less stringent than for Miss Roman's "Catalogue", it includes 1,315 entries-approximately twice the number in the more selective northern list. Tangential velocities were computed for all stars south * Memoirs of the Mount Stromlo Observatory. No. 14 (Fourth Number of Vol. 3): Southern Stars of High Velocity $-a$ fnding list compiled by william Buscombe and Pamela M. Morris. Pp. 36. compiled by William Buscombe and Pamela M.
(Canberra : Australian National University, 1958.) 